\title{
One-day nasogastric tube decompression after distal gastrectomy: a prospective randomized study
}

\author{
Alberto Mangano ${ }^{1} \cdot$ Laura Marciano $^{2}$
}

Received: 15 May 2017 / Accepted: 5 June 2017 / Published online: 19 July 2017

(C) Springer Japan KK 2017

Keywords Distal gastrectomy · Nasogastric tube · Evidence based surgery $\cdot$ Randomized controlled trial

Sir,

We do appreciate the aim of the prospective, randomized trial conducted by Kimura et al. [1], to assess whether standard 1-day nasogastric tube (NGT) decompression is an avoidable step in distal gastrectomy. De facto, distal gastrectomy is a common operation, particularly in Asia, with a not uncommon complication rate. Hence, we read this valuable manuscript with extreme interest. We agree with the authors that at this stage, the scientific literature has not clarified whether the uncomfortable procedure of 1-day NGT placement is of clinical benefit in preventing postoperative complications. For this reason, if the aforementioned question is answered, we could theoretically avoid inflicting the discomfort of NGT decompression on patients undergoing distal gastrectomy. In their conclusion, our colleagues claim that there were no significant postoperative complications or postoperative course recovery differences between the NGT and non-NGT arms of their series. However, despite using appropriate statistical tests, it does not seem that they have calculated a pre-hoc sample size. In other words, whenever a prospective study is

This comment refers to the article available at doi:10.1007/s00595-017-1475-0.

Alberto Mangano

alberto.mangano@gmail.com

Via Mulini 12, 22015 Gravedona ed Uniti (Como), Italy

2 University Center of Statistics for the Biomedical Sciences, Vita-Salute San Raffaele University, Via Olgettina 58, 20132 Milan, Italy conducted, it is essential to preliminarily establish the number of individuals needed to reach sufficient statistical power. In particular, the sample size calculation is influenced by the frequency of occurrence of the phenomenon we want to test; that is, the lower the occurrence, the larger the sample size needed to achieve sufficient statistical power. Moreover, the sequence allocation method was not specified and this is another relevant element that must be mentioned when performing a randomized trial [2]. When a new approach or surgical technique (or variation of a pre-existing protocol) is introduced, it is important that it is accurately standardized to achieve reproducible results among different centers [3]. Hence, it would be interesting to conduct additional prospective multicenter trials in the same area to confirm the authors' interesting conclusions and add further robust and statistically based data.

Again, we thank our colleagues for their strong contribution toward a better understanding of this topic.

Compliance with ethical standards

Conflict of interest The authors declare that they have no competing interests.

\section{References}

1. Kimura Y, Yano H, Iwazawa T, Fujita J, Fujita S, Yamamoto K, Yasuda T. One-day nasogastric tube decompression after distal gastrectomy: a prospective randomized study. Surg Today. 2017. doi:10.1007/s00595-017-1475-0.

2. Dettori J. The random allocation process: two things you need to know. Evid Based Spine Care J. 2010;1(03):7-9.

3. Mangano A, Rausei S, Lianos GD, Dionigi G. Quality of life after gastrectomy for adenocarcinoma: a prospective cohort study. Ann Surg. 2015;262(6):e110. 